\title{
Kinematic and dynamic analysis of planetary mechanisms with two degrees of freedom used for power sources coupling
}

\author{
I Tudor, B Florescu \\ University of Piteşti, Street Târgul din Vale 1, Piteşti 110040, România \\ "Corresponding author e-mail irina_diaconu@live.com \\ Article history \\ Received 10.05.2017 \\ Accepted 23.05.2017 \\ DOI https://doi.org/10.26825/bup.ar.2017.014
}

\begin{abstract}
In this paper we perform a review of the planetary gear trains with two central gears, and a planet carrier, used for power sources coupling. The kinematic and dynamic analysis was determined for a simple planetary gear mechanism, with two degrees of freedom formed by two gears that are in external meshing. For the same planetary mechanism, it was determined the mechanical efficiency and the associated internal powerflow direction, which helps identify the planetary gear train with the highest mechanical efficiency.
\end{abstract}

\section{Introduction}

The planetary mechanisms are used in hybrid gear train transmissions because they offer several advantages: can achieve high gear ratios at small dimensions, have a compact construction, can achieve gear ratio coupling without interrupting the power-flow. [1].

There is a wide variety of planetary mechanisms, but choosing the appropriate planetary mechanism, is based on its transmission ratio, size, and mechanical efficiency.

The most commonly used planetary mechanisms for coupling power sources are the planetary mechanisms 2K-H [1]. This description was made by Professor N. V. Kudreaţev, and according to his theory, the sun gears are marked with the letter $\mathrm{K}$ and the planet carrier with the letter $\mathrm{H}$.

Due to their small sizes and weights, the planetary gear trains with two sun gears and a planet carrier are frequently used for power sources coupling. They also have the ability to transmit a wide range of power values. They can operate at powers as low as a few watts up to many kilowatts. 
Whenever there is the need to choose the driving mechanism, it important to take into consideration the fact that the planetary mechanisms have a greater design complexity than the ordinary gear trains [1].

The technical performances of a planetary mechanism are not the only reasons for which they are so appreciated in worldwide markets, the mechanical efficiency being one of its most important characteristic.

When designing a planetary gear train, a special attention is paid to determining the mechanical efficiency.

\section{Kinematic structures of planetary mechanisms with two central gears and a planet} carrier used for power sources coupling

The planetary mechanisms with two central gears and a planet carrier offer a wide variety of design solutions: single stage planetary mechanism (figure.1 and figure 3) or two stages planetary mechanisms (figure 2, figure 4, and figure 5) [1].

Taking into consideration the gear mesh, the planetary mechanisms can have internal and external meshing.

The single stage bevel gear mechanism showed in figure 3 is typically used as a gear reducer for automobiles transmissions, machine tools and various devices. [2].

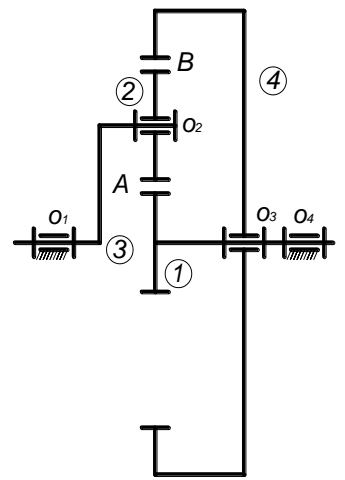

Figure 1. Planetary mechanism $2 \mathrm{~K}-\mathrm{H}$ with an internal and an external meshing.

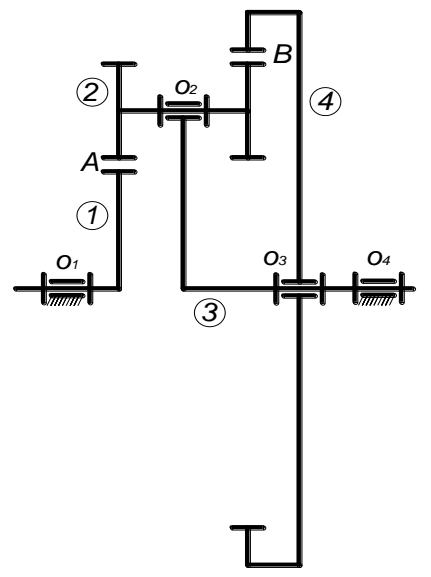

Figure 4. Planetary mechanism $2 \mathrm{~K}-\mathrm{H}$ with a double satellite, external and internal meshing
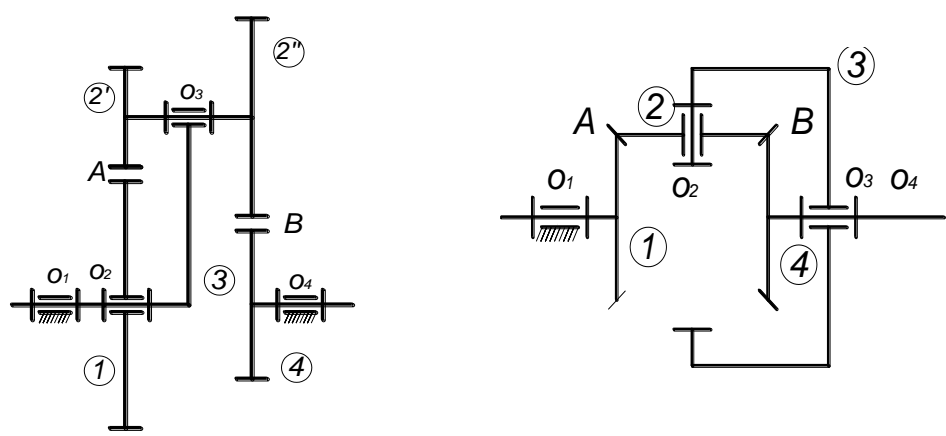

Figure 2. b Planetary mechanism $2 \mathrm{~K}-\mathrm{H}$ with a double

satellite and two eternal meshing
Figure 3. Planetary mechanism $2 \mathrm{~K}-\mathrm{H}$ differential.
Figure 5. Planetary mechanism $2 \mathrm{~K}-\mathrm{H}$ with a double satellite, and two internal meshing 
To better understand the way that planetary mechanisms $2 \mathrm{~K}-\mathrm{H}$ work, we started by determining the kinematic and dynamic analysis for a basic planetary gear mechanism with two degrees of freedom.

\section{Kinematic analysis for basic planetary mechanism with two degrees of freedom}

To determine the characteristics of the planetary mechanisms presented above, we begin by describing the basic planetary gear mechanism with external meshing and two degrees of freedom [4].

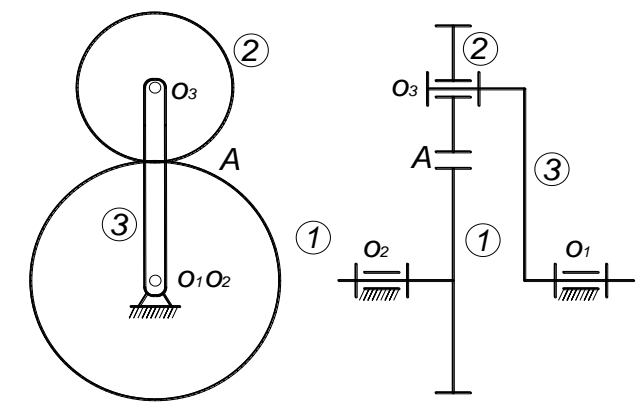

Figure 6. Basic planetary gear mechanism with

external meshing and two degrees of freedom

The basic planetary gear mechanism showed in figure 6, consists of the central gear 1 with the radius $r_{1}$, and the satellite 2, whose radius is denoted $r_{2}$. The gear's 2 axis is the axis for the mobile crank 3 , also called a planet carrier.

To determine the planetary gear trains ratio there are three proposed methods [1]:

a) the first one, the Willis method also called the inverted motion method, it transforms the planetary gear train into an ordinary one [1], [4], [5].

b) the second one is the decomposition method, also called the Swamp method, which decomposes the complex movements into simple ones [1], [4], [5].

c) the graphic-analytical method is also known as the instantaneous centers of velocity method [1].

All three methods are based on the principle that the speed of any planetary mechanisms element is equal to the speed of another element to which you add their relative speed.

Of the three listed above, the most commonly used method is the Willis method.

For basic planetary mechanism with two degrees of freedom, shown in figure 6 , we shall consider the mechanism gear ratio $i_{12}^{3}$.

$$
i_{12}^{3}=-\frac{z_{1}}{z_{2}},
$$

where $z_{1}$ and $z_{2}$ is gear 1 and 2 teeth numbers.

the ratio $i_{12}^{3}$ can have positive value if the two meshing gears are in external mesh, or negative value, if they are in internal mesh. In this case, the gear ratio has a positive value because the two gears are in external mesh.

According to Willis's theory:

which lead to:

$$
i_{12}^{3}=\frac{\omega_{1}-\omega_{3}}{\omega_{2}-\omega_{3}}
$$

$$
\omega_{1}-\omega_{2} i_{12}^{3}-\left(1-i_{12}^{3}\right) \omega_{3}=0 .
$$

It is considered that the planetary mechanism rotates with a constant speed, and that all losses are negligible, except meshing losses.

4. Torque and associated power flow analysis of the basic planetary mechanism with external meshing and two degrees of freedom 
To determine the mechanical efficiency, it will be taken into account both friction and meshing losses [6], [9].

3.

We denote $M_{1}, M_{2}, M_{3}$, and $P_{1}, P_{2}, P_{3}$, the torques, respectively the powers applied to links 1,2 ,

If the planetary mechanism is in equilibrium then:

$$
\begin{gathered}
M_{1}+M_{2}+M_{3}=0, \\
P_{1}+P_{2}+P_{3}+P_{F}=0,
\end{gathered}
$$

where $P_{F}$ represents the meshing and friction power losses.

The value of $\mathrm{P}_{\mathrm{F}}$ does not modify, if the entire mechanism is rotated with an angular velocity equal, but negative in value to the planet carriers angular velocity. This way, the mechanism turns into a gear mechanism with fixed axis, also called an ordinary gear train [1], [6], [7].

To determine the power losses it is necessary to establish the mechanisms input link. [5]:

The basic planetary mechanism, powers are determined with the following expressions [1], [4],

$$
\begin{gathered}
P_{1}=M_{1}\left(\omega_{1}-\omega_{3}\right), \\
P_{2}=M_{2}\left(\omega_{2}-\omega_{3}\right), \\
P_{3}=M_{3}\left(\omega_{3}-\omega_{3}\right)=0 .
\end{gathered}
$$

Just as the central gear 1 and the satellite 2 are either input link or the driven link, the power $P_{1}$ respectively $P_{2}$ applied to these elements, have positive or negative values.

By replacing into the equation (5) one obtains:

$$
P_{F}=-M_{1}\left(\omega_{1}-\omega_{3}\right)-M_{2}\left(\omega_{2}-\omega_{3}\right) \text {. }
$$

\section{The mechanical efficiency and the power flow direction of the basic planetary mechanism with external meshing and two degrees of freedom}

Because of power losses we define the mechanical efficiency of the planetary mechanism, as being the ratio between the output power, and the power applied at the input link[2].

$$
\begin{gathered}
\eta=\frac{P_{o}}{P_{m}}=-\frac{M_{0} \omega_{0}}{M_{m} \omega_{m}}, \\
P_{o}=P_{m}-P_{F},
\end{gathered}
$$

where:

$\mathrm{P}_{\mathrm{o}}, \mathrm{P}_{\mathrm{m}}$ is denoted as the output power, respectively, the input power for the planetary mechanism,

$M_{m}, \omega_{m}$, represents the torque and the angular velocity of the input shaft,

$M_{0}, \omega_{0}$, represents the torque and the angular velocity of the output shaft.

To determine the mechanical efficiency for the basic planetary gear mechanism, we will use the inverted motion method, this method being the most commonly used of the three listed above.

It was determined the efficiency of the basic planetary mechanism shown in figure 6 for both cases when the input link is either gear 1 or gear 2.

Let us consider gear 1 as the input link of the basic planetary mechanism shown in figure 6 [6], [8]. Under the kinematic inversion the planetary mechanism, rotates with an angular speed equal and in opposite direction to the planet carrier's angular velocity. In this situation the planet carrier is fixed and the planetary mechanism becomes an ordinary gear train and helps us determine the efficiency with the following expression:

According to equation (10):

$$
\eta=-\frac{M_{2}\left(\omega_{2}-\omega_{3}\right)}{M_{1}\left(\omega_{1}-\omega_{3}\right)}
$$

where $M_{1}\left(\omega_{1}-\omega_{3}\right)$ is the input power; 


$$
\begin{aligned}
& M_{1}\left(\omega_{1}-\omega_{3}\right)>0 \text { because } M_{1}>0 \text { and }\left(\omega_{1}-\omega_{3}\right)>0 \\
& M_{2}\left(\omega_{2}-\omega_{3}\right) \text { is the output power. }
\end{aligned}
$$

By substituting according to relation (2) one obtains:

$$
M_{1} \eta i_{12}^{3}+M_{2}=0 \text {. }
$$

If we consider gear 2 as the input link of the basic planetary mechanism shown in figure 6 under the kinematic inversion, the planetary efficiency is: [6], [8]:

where $M_{2}\left(\omega_{2}-\omega_{3}\right)$ is the input power,

$$
\eta=-\frac{M_{1}\left(\omega_{1}-\omega_{3}\right)}{M_{2}\left(\omega_{2}-\omega_{3}\right)},
$$

$M_{2}\left(\omega_{2}-\omega_{3}\right)>0$ because $M_{2}>0$ and $\left(\omega_{2}-\omega_{3}\right)>0$;

$M_{1}\left(\omega_{1}-\omega_{3}\right)$ is the output power.

By substituting according to relation (2) one obtains:

$$
\frac{M_{1} i_{12}^{3}}{\eta}+M_{2}=0 .
$$

The expressions (13) and (15) are known as the basic equations for determining the mechanical efficiency of a basic planetary gear mechanism with two degrees of freedom. They can be taken into account simultaneously by defining the parameter $\alpha$

considering:

$$
\alpha=\left\{\begin{array}{c}
1, \text { if the grear } 1 \text { is the input link } \\
-1, \text { if the grear } 2 \text { is the input link }
\end{array}\right\} .
$$

We can define the basic torque equation for the planetary mechanism with two degrees of freedom which depends on the mechanical efficiency:

$$
M_{2}+M_{1} i_{12}^{3} \eta^{\alpha}=0
$$

\section{Kinematics analyses for $2 \mathrm{~K}-\mathrm{H}$ single row planetary mechanism with external and internal meshing}

The most commonly used mechanism for coupling power sources is the $2 \mathrm{~K}-\mathrm{H}$ single row planetary mechanism with an external - internal mesh.

This solution was initially adopted and later on perfected by the Japanese manufacturer Toyota, to be fitted on the hybrid model Prius. The brand name for the hybrid drive train technology was Toyota Hybrid System.

The planetary mechanism composed of the two central gears and a planet carrier that are in external and internal mesh, it is the main component of the Toyota Hybrid System. The kinematic scheme for this planetary mechanism is described in figure 1

We have denoted $z_{1}, z_{2}, z_{4}$, the number of teeth for the gears $1,2,4$.

With $\omega_{1}, \omega_{2}, \omega_{4}$ it is denoted the absolute angular velocities of satellite 2 , sun gear 1 and the ring gear 4 . If we consider for the mechanism described in figure 2 , the parameter $\omega_{3}$, as being the absolute angular velocity of the planet-carrier 3, one can obtain [6], [8]:

that leads to:

$$
\begin{aligned}
& \frac{\omega_{1}-\omega_{3}}{\omega_{2}-\omega_{3}}=-\frac{z_{2}}{z_{1}}=i_{12}^{3}, \\
& \frac{\omega_{1}-\omega_{3}}{\omega_{4}-\omega_{3}}=-\frac{z_{4}}{z_{1}}=i_{14}^{3}, \\
& \frac{\omega_{2}-\omega_{3}}{\omega_{4}-\omega_{3}}=\frac{z_{4}}{z_{2}}=i_{42}^{3},
\end{aligned}
$$

from which we obtain the following equation:

$$
\begin{aligned}
& \omega_{2}-i_{12}^{3} \omega_{1}+\left(i_{12}^{3}-1\right) \omega_{3}=0, \\
& \omega_{2}-i_{42}^{3} \omega_{4}+\left(i_{42}^{3}-1\right) \omega_{3}=0, \\
& \omega_{1}-i_{14}^{3} \omega_{4}+\left(i_{14}^{3}-1\right) \omega_{3}=0 .
\end{aligned}
$$

\section{The mechanical efficiency and the power - flow direction for the planetary mechanism}


Using the judgment described in the first part of the paper, the kinematic analysis for the planetary mechanism with two central gears and a planet carrier with external and an internal mesh is determined. [2].

The mechanical efficiency expressions for this planetary mechanism are [2], [4], [6], [8], [9]:

Table 1. Mechanical efficiency for $\alpha>0$.

\begin{tabular}{|c|c|c|c|}
\hline \multirow{2}{*}{ Input link } & \multirow{2}{*}{$\begin{array}{c}\text { Driven } \\
\text { link }\end{array}$} & \multicolumn{2}{|l|}{ Mechanical Efficiency } \\
\hline & & $\alpha>0$ & \\
\hline $1+4$ & 3 & $\frac{\left(1-\frac{i_{14}^{3}}{\eta_{14^{3}}}\right)\left(i_{14}-i_{14}^{3}\right)}{\left(1-i_{14}^{3}\right)\left(i_{14}-\frac{i_{14}^{3}}{\eta_{14^{3}}}\right)}$ & (24) \\
\hline $1+3$ & 4 & $\frac{i_{14}^{3} \eta_{14^{3}}\left(1-i_{14}^{3}\right)}{\left(1-i_{14}^{3}\right) i_{14}-\left(1-i_{14}^{3} \eta_{14^{3}}\right)\left(i_{14}-i_{14}^{3}\right)}$ & (35) \\
\hline $4+3$ & 1 & $\frac{i_{14}\left(1-i_{14}^{3}\right)}{i_{14}^{3} \eta_{14^{3}}\left(1-i_{14}^{3}\right)+\left(1-i_{14}^{3} \eta_{14^{3}}\right)\left(i_{14}-i_{14}^{3}\right)}$ & (46) \\
\hline 3 & $1+4$ & $\frac{\left(i_{14}-\frac{i_{14}^{3}}{\eta_{14^{3}}}\right)\left(1-i_{14}^{3}\right)}{\left(i_{14}-i_{14}^{3}\right)\left(1-\frac{i_{14}^{3}}{\eta_{14^{3}}}\right)}$ & (58) \\
\hline 4 & $1+3$ & $\frac{i_{14}\left(1-i_{14}^{3}\right)-\left(1-i_{14}^{3} \eta_{14^{3}}\right)\left(i_{14}-i_{14}^{3}\right)}{i_{14}^{3} \eta_{14^{3}}\left(1-i_{14}^{3}\right)}$ & (29) \\
\hline 1 & $4+3$ & $\frac{i_{14}^{3} \eta_{14^{3}}\left(1-i_{14}^{3}\right)+\left(1-i_{14}^{3} \eta_{14^{3}}\right)\left(i_{14}-i_{14}^{3}\right)}{i_{14}\left(1-i_{14}^{3}\right)}$ & (30) \\
\hline
\end{tabular}

Table 2. Mechanical efficiency for $\alpha<0$.

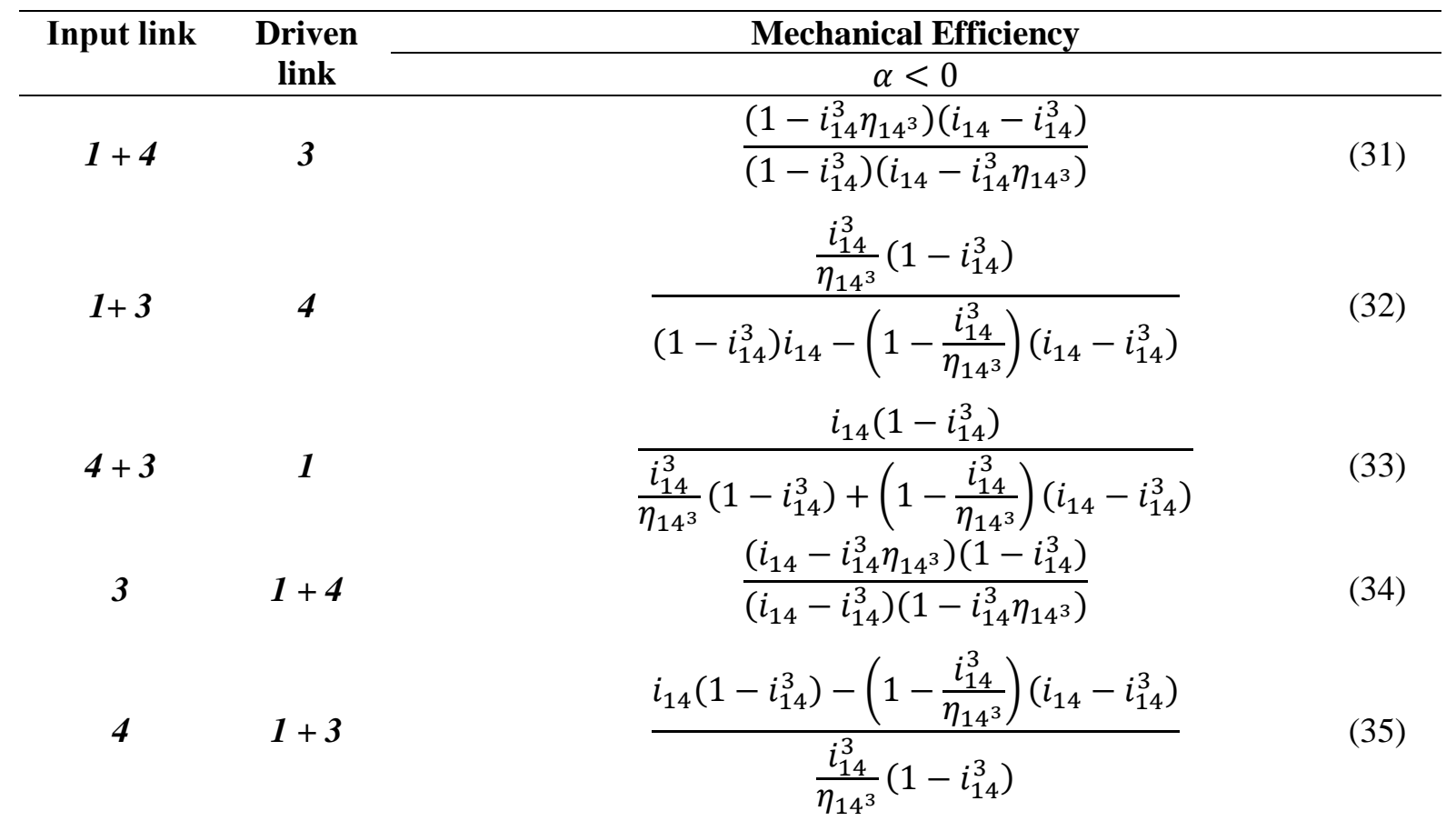




$$
14+3 \frac{i_{14}\left(1-i_{14}^{3}\right)-\left(1-\frac{i_{14}^{3}}{\eta_{14^{3}}}\right)\left(i_{14}-i_{14}^{3}\right)}{\frac{i_{14}^{3}}{\eta_{14^{3}}}\left(1-i_{14}^{3}\right)}
$$

where: $\quad \eta_{14}^{3}=$ the gears internal efficiency;

$$
i_{14}^{3}=-\frac{z_{4}}{z_{1}}=\text { the gear ratio. }
$$

The parameter $\alpha$ is associated to the gear mechanical efficiency in the power equilibrium equation for the ordinary gear train. This parameter specifies if the element 1 is an input link or a driven link in the inversed planetary mechanism.

It can have a negative or a positive value, and [2] can be determined with the following relation:

$$
\alpha=\operatorname{sign}\left[\frac{M_{1}\left(\omega_{1}-\omega_{3}\right)}{M_{1} \omega_{1}}\right]=\operatorname{sign} \frac{i_{14}^{3}\left(1-i_{14}\right)}{i_{14}\left(1-i_{14}^{3}\right)},
$$

Numerical example:

To determine the mechanical efficiency for the single row planetary mechanism with external and internal mesh showed in figure 1 , we consider $z_{1}=22$ teeth, $z_{2}=18$ teeth, $z_{4}=58$ teeth, $i_{34}=2$ and $\eta_{14}^{3}=0,95$.

According to equation (38):

$$
i_{14}^{3}=-\frac{z_{4}}{z_{1}}=-\frac{58}{22}=-2,636,
$$

To determine the mechanical efficiency it is necessary to first calculate the parameter $\alpha$ By substituting in equation (39) one obtains:

$$
\begin{gathered}
\alpha=\mp \operatorname{sign} \frac{i_{14}^{3}\left(1-i_{14}\right)}{i_{14}\left(1-i_{14}^{3}\right)}=\frac{-2,636 \cdot(1-2)}{2 \cdot(1-2,636)}=\frac{2,636}{-3,272}=-0,8056 \\
<0 .
\end{gathered}
$$

By substituting into the equations presented in the table 2, for the single row planetary mechanism with external and internal meshing, we have obtained the following results:

Table 3. Numerical results.

\begin{tabular}{ccc}
\hline Input link & Driven link & Mechanical efficiency \\
\cline { 3 - 3 } & & $\alpha<0$ \\
$4+3$ & 1 & $\eta=0,992$ \\
$\mathbf{1}+\mathbf{1}$ & $\mathbf{3}$ & $\eta=0,986$ \\
$\mathbf{4}$ & $\mathbf{1}$ & $\eta=0,981$ \\
$\mathbf{3}$ & $\mathbf{1 + 3}$ & $\eta=0,992$ \\
$\mathbf{1}$ & $\mathbf{1 + 3}$ & $\eta=0,986$ \\
& $\mathbf{4}$ & $\eta=0,982$ \\
\hline
\end{tabular}

\section{Conclusions}

Based on the judgment used for expressing the kinematic and dynamic analysis of a basic planetary mechanism with two degrees of freedom, it has been determined the mechanical efficiency for the planetary mechanisms with two central gears and a planet carrier. Because, in addition to the reduced dimensions, the manufacture, assembly and operation requirements, the mechanical 
efficiency is a very important feature for the planetary mechanisms, it is very important to correctly determine it.

Due to higher efficiency values one can conclude that the most commonly used planetary mechanism in power sources coupling is the single row planetary mechanism with external and internal mesh (figure 1). In addition to the fact that is has high efficiency values, this mechanism has also a simple assembly condition, as opposed the planetary mechanism with a double satellite and interior meshing showed in (figure 5).

Usually used as a differential reducer in the automotive industry, machine tools and various devices, the differential mechanism showed in figure 3, provides high efficiency values [1].

The planetary mechanism with a double satellite and external meshing presented in (figure 2) can be characterized by high gear ratios and small efficiency values, and it is not recommended to for equipping power transmissions.

\section{References}

[1] Bostan I, Dulgheru V and Grigoraș S, Transmisii planetare precesionale şi armonice, 1997, ed Atlas Tehnică Bucureşti, ed. Tehnică UTM,

[2] Miloiu G and Dudiță F, Transmisii mecanice moderne, 1971, ed. Tehnica Bucureși,

[3] Simionescu I and Moise V, Mecanisme, 1999, ed. Tehnică București,

[4] Cheng W and Huan Y, C, The analysis of power circulation and the simplified expression of the transmission efficiency of 2K-H closed epicycic gear trains, 2013, Meccanica,

[5] Artde D K T Lam, A study on the Mechanical efficiency of Power Split Unit of Toyota Hybrid System, 2011, J. of Scie. and Innov. Vol 1, Nr. 4,

[6] Pennestrì E, and Valentini P P, A review of formulas for mechanical efficiency analysis of two degrees of freedom epyciclyc gear train, 2003, Mech, Eng.,

[7] Pennestrì E, and Valentini P P, Dynamic analysis of epicyclic gear trains by means of computer algebra, 2002, Kluwer Academic Publishers,

[8] Mantriota G, Pennestrì E, Theoretical and experimental efficiency analysis of multi-degrees-offreedom epicyclic gear trains, 2003, Kluwer Academic Publishers,

[9] Cho S, Ahn K, Lee J M, Efficiency of the planetary gear hybrid powertrain, 2005, School of Mechanical and Aerospace Engineering, Seoul National University, Republic of Korea,

[10] Petrovic $\mathrm{T}$, Ivanov $\mathrm{T}$ and Milosevic $\mathrm{M}$, A new structure of combined gear trains with high transmission ratios, 2009, Forsch Ingenieurwes,

[11] Yang H, Kim B, Park Y, Lim W, and Cha S, Analysis of planetary gear hybrid powertrain system part 2: output split system, 2009, Int, J. of Autom. Tech., 